\title{
A ATENÇÃO À PACIENTES COM HIV/AIDS E OS CUIDADOS DE ENFERMAGEM PARA PROMOÇÃO DA QUALIDADE DE VIDA
}

\author{
CARE FOR PATIENTS WITH HIV/AIDS AND NURSING CARE TO PROMOTE \\ QUALITY OF LIFE
}

\author{
Kehetellen Ellen Barbosa dos Santos ${ }^{1}$ \\ Tamires Ribeiro Santos ${ }^{2}$ \\ Camila Silva e Souza ${ }^{3}$
}

RESUMO: Introdução: A atuação do enfermeiro para promoção da qualidade de vida do paciente ocorre nas mais variadas doenças e patologias, destaca-se neste trabalho a prática direcionada ao paciente com HIV/AIDS perpassando pelos aspectos técnicos até se chegar de fato ao tratamento paliativo efetivo. Objetivo: Compreender o processo que leva o sujeito infectado com o HIV/AIDS a ter qualidade de vida a partir dos aspectos físicos, psicossocial e emocional no atendimento oferecido pelo SUS com a efetivação dos Direitos fundamentais através do enfermeiro. Material e Métodos: Trata-se de uma revisão integrativa de literatura, as bases de dados utilizadas são: NCBI/PubMed (National Center for BiotechnologyInformation), SciELO (Scientific Eletronic Lirary Online), Google Acadêmico e Lilacs - Bireme (Literatura Latino-Americana e do Caribe em Ciências da Saúde). Para delimitação dos conteúdos foram utilizados critérios de inclusão: artigos disponíveis na integra, em português e inglês, publicados no período de 2014 a 2021 com acesso gratuito com pertinência temática, ao final foram selecionados io artigos para análise. Resultados e discussão: $O$ consenso de que a qualidade de vida existe a partir da desenvoltura de toda uma engrenagem constituída pela efetivação dos direitos fundamentais pelo Estado perante as pessoas que vivem com o HIV/AIDS através da oferta de tratamentos e medicamentos que atendam às suas necessidades a ponto de possibilitar o acesso à saúde concretizando o direito ligado à dignidade humana, ressaltando, sobretudo a assistência do enfermeiro neste contexto do paciente com HIV/AIDS. Considerações finais: Conclui-se que o enfermeiro deve atuar no âmbito dos pacientes com HIV/AIDS com tecnicidade, respeito, dignidade, ética, profissionalismo, tratando paciente em pé de igualdade e esclarecendo dentro de seus conhecimentos os principais direitos correlacionados ao atendimento no sistema de saúde.

Palavras-chave: Qualidade de Vida. SUS. Enfermagem. HIV/AIDS.

\footnotetext{
${ }^{I}$ ORCID: https://orcid.org/oooo-ooor-9825-550o. Lattes: http://lattes.cnpq.br/4248074033040698. Faculdade Integrada Carajás (FIC). E-mail:kehetellen202i@gmail.com.

2 ORCID: https://orcid.org/oooo-ooo3-1020-7163. Lattes: http://lattes.cnpq.br/o727883188883203 Faculdade Integrada Carajás (FIC). E-mail:tamipresribeiro984@gmail.com

3 ORCID: https://orcid.org/oooo-ooor-9865-5299. Lattes: http://lattes.cnpq.br/6534066790821014. Faculdade Integrada Carajás (FIC).E-mail:prof.camilasilvasouza@outlook.com
} 
ABSTRACT: Introduction: The role of the nurse to promote the quality of life of the patient occurs in the most varied diseases and pathologies, this work highlights the practice directed to the patient with HIV/AIDS, passing through the technical aspects until reaching the effective palliative treatment. Objective: Understand the process that leads the subject infected with HIV/AIDS to have quality of life based on the physical, psychosocial and emotional aspects of the care offered by the SUS with the realization of Fundamental Rights through the nurse. Material and Methods: This is an integrative literature review, the databases used are: NCBI/PubMed (National Center for BiotechnologyInformation), SciELO (Scientific Electronic Lirary Online), Academic Google and Lilacs - Bireme (Latin American Literature and the Caribbean in Health Sciences). To delimit the contents, inclusion criteria were used: articles available in full, in Portuguese and English, published from 2014 to 2021 with free access with thematic relevance, in the end, io articles were selected for analysis. Results and discussion: The consensus that quality of life exists based on the resourcefulness of a whole gear constituted by the realization of fundamental rights by the State towards people living with HIV/AIDS through the provision of treatments and medications that meet their needs to the point of enabling access to health, fulfilling the right linked to human dignity, emphasizing, above all, the care provided by nurses in this context of patients with HIV/AIDS. Final considerations: It is concluded that nurses should work with HIV/AIDS patients with technicality, respect, dignity, ethics, professionalism, treating patients on an equal footing and clarifying within their knowledge the main rights correlated to care in the system of health.

Keywords: Quality of life. SUS. Nursing. HIV/AIDS.

\section{INTRODUÇÃO}

A atenção à pacientes com HIV/AIDS e os cuidados de enfermagem para promoção da qualidade de vida, são fundamentais para realização de um atendimento de qualidade direcionado ao paciente, ainda que sede de cuidados paliativos e por compreender que o HIV/AIDS não possui cura, o acompanhamento por equipe médica é necessário, e o enfermeiro deve estar na linha de frente para conscientização e retirada de dúvida (HIPÓLITO et al., 2017).

Dentre os principais aspectos da necessidade de contato com equipe médica é para promoção da qualidade de vida, isso porque esta independe de tratamento médico. É plausível frisar que a AIDS não afeta somente o físico do ser humano, mas também o convívio social e o sistema emocional, visto que existem muitos preconceitos na sociedade com as pessoas infectadas, por isso, a necessidade de discutir e refletir sobre a qualidade de vida destas pessoas, para que as mesmas não sejam excluídas das atividades laborais, de lazer e enfim da liberdade e igualdade (MAIA; REIS JÚNIOR, 2019). 
Estudar e refletir sobre a condição de vida das pessoas portadoras do HIV/AIDS é necessário, uma vez que o enfermeiro precisa saber lidar com as enfermidades que fazem parte do cotidiano hospitalar, e ainda deve-se levar em consideração o pré-conceito e descrédito dessa população no âmbito da sociedade, não sendo admissível que $s$ enfermeiros enquanto profissionais de saúde juramentados não tenham tecnicidade e tato no tratamento e assistência ao paciente com HIV/AIDS (OLIVEIRA, 2019).

Assim, a escolha deste tema surge na necessidade de compreender atuação do enfermeiro para promoção da qualidade de vida do paciente infectado, e ainda destacar que qualquer patologia requer tratamento e o olhar de assistência e empatia do profissional de saúde, o qual é crucial por ser esta uma das funções e finalidades dos enfermeiros e enfermeiras atuantes em unidades hospitalares ou qualquer ambiente em que o enfermeiro é necessário, visando um atendimento integral e humanizado (OLIVEIRA et al., 2015).

Assim, o objetivo deste trabalho é compreender o processo que leva o sujeito infectado com o HIV/AIDS a ter qualidade de vida a partir dos aspectos físicos, psicossocial e emocional no atendimento oferecido pelo SUS com a efetivação dos Direitos fundamentais através do enfermeiro.

\section{MATERIAIS E MÉTODOS}

Para fundamentar o presente estudo utilizou-se o método de revisão integrativa bibliográfica de modo a apresentar os trabalhos pertinentes $\mathrm{A}$ atenção à pacientes com HIV/AIDS e os cuidados de enfermagem para promoção da qualidade de vida. Para validação da bibliografia utilizada as pesquisas foram realizadas nas seguintes bases de dados eletrônicas: NCBI/PubMed (National Center for BiotechnologyInformation), SciELO (Scientific Eletronic Lirary Online) e Lilacs - Bireme (Literatura LatinoAmericana e do Caribe em Ciências da Saúde), com os seguintes descritores "Qualidade de Vida"; "SUS"; "Enfermagem"; "HIV/AIDS”, e seus respectivos correspondentes em inglês.

A partir desse conjunto de palavras-chave e para a busca dos artigos, através dos filtros das próprias bases de dados, estabeleceram-se os seguintes critérios de inclusão: artigos disponíveis na integra, em português e inglês, no período de 2014 a 2021 com acesso gratuito e que tivessem afinidade com a temática. Estabeleceu-se ainda, os tipos de estudos 
aceitos revisão bibliográfica, sistemática, integrativa, relato de experiência, estudo transversal e foram excluídos os manuscritos repetidos ou duplicados fora do período definido para o estudo e sem adequação aos objetivos da pesquisa.

\section{RESULTADOS}

Para construção do aspecto teórico deste trabalho foram aplicadas as etapas de pesquisa através dos descritores, encontrando 64 artigos sobre o tema escolhido, após aplicação dos critérios de inclusão e exclusão foram selecionados io artigos para análise. As plataformas de pesquisa utilizadas são as seguintes: PubMed, SciELO, Google Acadêmico (G.A) e Lilacs. Os resultados apresentados acima foram dispostos no Fluxograma I.

Fluxograma r: Fluxograma das etapas de inclusão e exclusão dos artigos.
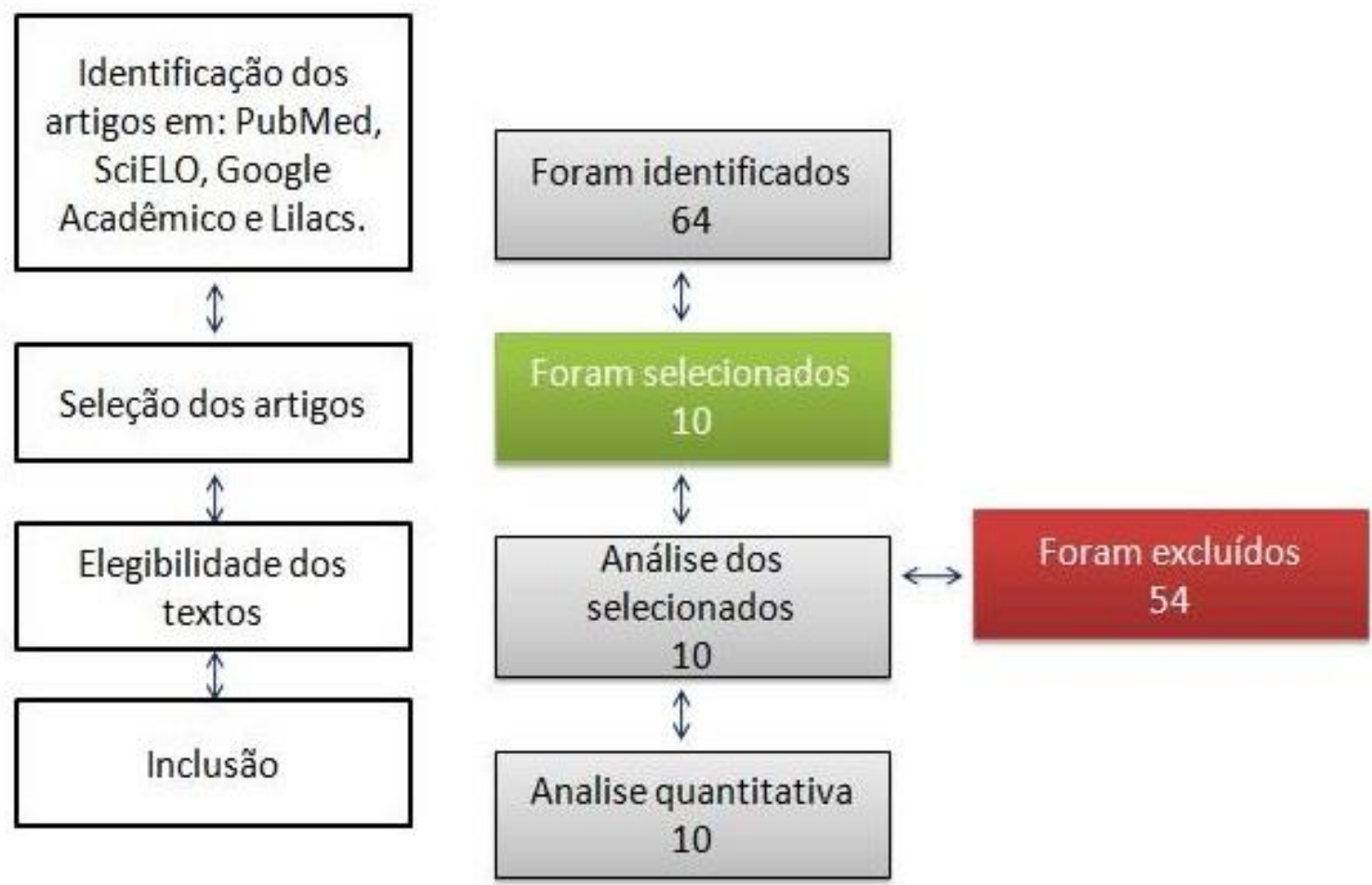

Fonte: Santos; Santos; Souza (2021).

Após a análise dos artigos foram selecionados ıo estudos produções para integrar este artigo de revisão. A Tabela I apresenta os textos escolhidos e sua distribuição por autoria, ano de publicação, título, revista e base de dados dos estudos selecionados. 
Tabela r: Estudos selecionados segundo autoria, ano de publicação, título, revista e base de dados dos estudos selecionados.

\begin{tabular}{|c|c|c|c|c|c|c|}
\hline \multirow[b]{2}{*}{ Autor/Ano } & \multirow[b]{2}{*}{ Título } & \multirow[b]{2}{*}{ Revista } & \multicolumn{4}{|c|}{ Base de dados } \\
\hline & & & $\begin{array}{l}\text { Pub } \\
\text { Med }\end{array}$ & SciELO & $\begin{array}{l}\text { G. } \\
\text { A }\end{array}$ & Lilacs \\
\hline $\begin{array}{l}\text { OLIVEIRA } \\
\text { et al., (2015) }\end{array}$ & $\begin{array}{l}\text { Mendes et al. Judicialização da } \\
\text { saúde: para onde caminham as } \\
\text { produções científicas? }\end{array}$ & $\begin{array}{l}\text { Revista Saúde em } \\
\text { Debate }\end{array}$ & & or & & \\
\hline $\begin{array}{l}\text { COSTA; } \\
\text { OLIVEIRA } \\
\text { FORMOZ } \\
\text { O, (2015) }\end{array}$ & $\begin{array}{l}\text { O setor saúde nas representações } \\
\text { sociais do HIV/Aids e qualidade } \\
\text { de vida de pessoas soropositivas. }\end{array}$ & $\begin{array}{l}\text { Revista da Escola } \\
\text { Anna Nery }\end{array}$ & or & & & \\
\hline $\begin{array}{l}\text { PEREIRA et } \\
\text { al., (2016) }\end{array}$ & $\begin{array}{l}\text { Pacientes com HIV/Aids e risco } \\
\text { de ulcera: demandas de } \\
\text { enfermagem }\end{array}$ & $\begin{array}{l}\text { Revista Brasileira de } \\
\text { Enfermagem }\end{array}$ & & or & & \\
\hline $\begin{array}{l}\text { MARTINS } \\
\text { et al., (2016) }\end{array}$ & $\begin{array}{l}\text { Qualidade de vida na infeção } \\
\text { VIH: perfis segundo o modo de } \\
\text { transmissão. }\end{array}$ & $\begin{array}{l}\text { Revista de Psicologia } \\
\text { Saúde \& Doenças }\end{array}$ & & or & & \\
\hline $\begin{array}{l}\text { HIPÓLITO } \\
\text { et al., (2017) }\end{array}$ & $\begin{array}{l}\text { Qualidade de vida de pessoas } \\
\text { convivendo com HIV/AIDS: } \\
\text { relação temporal, sócio } \\
\text { demográfica e perspectiva da } \\
\text { saúde. }\end{array}$ & $\begin{array}{lr}\text { Revista } & \text { Latino } \\
\text { Americana } & \text { de } \\
\text { Enfermagem } & \end{array}$ & & or & & \\
\hline $\begin{array}{l}\text { BRANDÃ } \\
\mathrm{O} \text { et al., } \\
(2017)\end{array}$ & $\begin{array}{l}\text { Representações sociais da equipe } \\
\text { de enfermagem perante o } \\
\text { paciente com HIV/AIDS: uma } \\
\text { revisão integrativa }\end{array}$ & $\begin{array}{l}\text { Revista de } \\
\text { Enfermagem UFPE }\end{array}$ & & & or & \\
\hline $\begin{array}{l}\text { MAIA; } \\
\text { REIS } \\
\text { JÚNIOR } \\
\text { (2019) }\end{array}$ & $\begin{array}{l}\text { Modos de enfrentamento do } \\
\text { HIV/AIDS: direitos humanos, } \\
\text { vulnerabilidades e assistência à } \\
\text { saúde. }\end{array}$ & Revista NUFEN & & & & or \\
\hline $\begin{array}{l}\text { OLIVEIRA } \\
(2019)\end{array}$ & $\begin{array}{l}\mathrm{O} \text { que as pesquisas revelam } \\
\text { sobre a saúde no Brasil. }\end{array}$ & Revista Inovar & & & or & \\
\hline
\end{tabular}




\begin{tabular}{|c|c|c|c|c|}
\hline $\begin{array}{l}\text { NETO et } \\
\text { al., (2019) }\end{array}$ & $\begin{array}{l}\text { Qualidade de vida no contexto } \\
\text { de pacientes com hiv/aids: um } \\
\text { estudo comparativo }\end{array}$ & $\begin{array}{ll}\text { Revista Saúde } & \text { e } \\
\text { Pesquisa } & \text { da } \\
\text { UNICEUSMAR } & \end{array}$ & & OI \\
\hline $\begin{array}{l}\text { MARQUES } \\
\text { et al., (2020) }\end{array}$ & $\begin{array}{l}\text { Avaliação da qualidade de vida } \\
\text { de pessoas vivendo com } \\
\text { HIV/AIDS: revisão integrativa }\end{array}$ & $\begin{array}{l}\text { Revista Enfermagem } \\
\text { UERJ }\end{array}$ & OI & \\
\hline
\end{tabular}

Fonte: Santos; Santos; Souza (2021).

\section{DISCUSSÃO TEÓRICA}

\section{I HIV/AIDS UM IMPORTANTE PROBLEMA DE SAÚDE}

A Síndrome da Imunodeficiência Adquirida (AIDS) surgiu nos EUA (Estados Unidos da América) no ano de 198I, em pessoas adultas, especialmente do sexo masculino e homossexuais sendo estes em sua maioria habitantes de Nova York ou São Francisco, os quais apresentaram na época problemas de imunidade com doenças como pneumonia e sarcoma de Kaposi (BRASIL, 2017).

Mediante, estas ocorrências patológicas que os estudiosos da época perceberam que surgia uma doença diferente, nova, infecciosa e transmissível. Assim, no ano de 1983 surge a identificação de um agente etimológico ou retrovírus humano ou HIV-I, que antes era chamado de LAV e HTLV -III e no final da década de 8o detectou-se o segundo agente etimológico que também era outro retrovírus, que tinha relação ao HIV - I, nomeado como HIV - 2 (BRASIL, 2017; MARQUES et al., 2020).

A HIV/AIDS surge no Brasil no ano de 1983 em São Paulo, por meio de um jovem homossexual, que apresentava na época perda de peso, febre, presença de gânglios no corpo e tuberculose, e assim a doença se espalhou no território brasileiro. É importante saber que o sujeito infectado pelo HIV que não fazem tratamento apresenta uma grave disfunção do sistema imunológico (NETO et al., 2019).

Devido à queda da taxa de linfócitos $\mathrm{T} \mathrm{CD}_{4}$, então, em 1996 foi instituída no Brasil uma Terapia denominada “Terapia Antirretroviral (TARV)", o que por sua vez contribuiu para aumentar o tempo de vida dos pacientes e também a qualidade de vida deles, através da diminuição do índice de infecções oportunizadas pela baixa imunidade, logo alterando a história da naturalidade da doença (BRASIL, 20I9). 
Em meados de 2012, houve uma estimativa de mais de dois milhões de pessoas infectadas com o HIV em todo o planeta terra, destes havia quase trezentos mil menores de 15 anos. Nesta mesma época, o Brasil ficou em 2o lugar no índice de pessoas mais infectadas pelo HIV, com presença de pessoas doentes em todas as suas regiões, principalmente as mais jovens e essa situação impactou negativamente na economia brasileira porque a força ativa do trabalho foi afetada pela doença (COSTA; OLIVEIRA; FORMOZO, 2015).

Desta forma, surge a necessidade de compreender a relevância do tratamento ainda que paliativo direcionado aos pacientes com HIV/AIDS e como o enfermeiro atua nesse cenário, assim torna-se necessário compreender a perspectiva legal.

\subsection{DIREITO À SAÚDE DO PORTADOR DE HIV/AIDS}

A vida e a saúde das pessoas constituem bens de valor imensurável, não podendo ser tratados de forma banal pelo Poder Público. Sendo-lhes garantidos direitos esculpidos na Carta Magna, não pode a sociedade brasileira ficar omissa diante das situações evidenciadas na saúde dos Portadores de HIV/AIDS e em outras situações patológicas, como no caso da Pandemia atual da Covid 19 demonstrando a desvalorização do ser humano (MARTINS et al., 2016).

Conforme o método de categorização de quem vive e quem morre, no que se refere ao tratamento. Do contrário, é imprescindível frisar que todas as vidas importam, sejam elas representadas por corpos físicos frágeis ou fortes; negros ou brancos; pobres ou ricos; doentes ou saudáveis (NETO et al., 2019).

O Art. 196 afirma ser direito do cidadão e dever do estado a oferta de saúde pública e gratuita, com escopo de amenizar as doenças, oferecendo serviços que recuperem, protejam e promovam o bem-estar dos que dela necessitem. A Carta Magna frisa ainda, no seu Art. 197, a importância dos serviços de saúde pública, devendo ser dispostos pelo poder público conforme a lei, cabendo também a este a sua fiscalização e regulamentação (BRASIL, 2019).

A própria sociedade deve estar consciente de suas garantias, com fulcro nos direitos fundamentais nascidos com o advento de inúmeras lutas realizadas por indivíduos de diferentes nações. A aprovação em 1948 da Declaração Universal dos Direitos Humanos 
pela ONU (Organização das Nações Unidas), foi um ato solene que serviu de pressuposto para a promoção do respeito a direitos e liberdades, o que por sua vez serve de fundamento nas práticas do Estado e dos cidadãos (PEREIRA et al., 2016).

Todavia, é relevante evidenciar que muitos dos direitos e garantias não são realizados de forma plena no meio social, por isso a necessidade de requerê-los perante a justiça. Ressalta-se que a reivindicação pela saúde de qualidade ofertada pelo Estado teve maior ápice nos anos de 1990, com o advento do vírus HIV (AIDS), contexto em que grande número de pessoas buscava ser atendida, estando acometidas da doença. (OLIVEIRA, 2019).

Assim, para garantir que o Poder Executivo programasse políticas públicas que abarcassem plenamente o direito à saúde, garantindo eficácia e qualidade à gratuidade de medicamentos antirretrovirais para todos os cidadãos, foi criada a Lei de no. 9.313/96. A partir daí, abriram-se precedentes, pois as pessoas aprenderam a buscar a ajuda da Justiça no tocante a outras patologias (BRANDÃO et al., 2017).

A busca incansável pela efetivação da saúde pública é motivada pela Carta Magna brasileira, apresentando em seu arcabouço diverso direitos, desde a inviolabilidade à vida, garantida pelo Art. $5^{\circ}$; passando pelo direito à saúde no Art. 196, e por princípios basilares de uma sociedade igualitária e justa. Nesse contexto, a demanda crescente de patologias e de sujeitos acometidos por doenças crônicas, cirurgias, transplantes e outros, levando ao aumento desenfreado por demanda judicial (BRASIL, 2017).

Assim, o paciente com HIV/AIDS necessita além do atendimento, precisa da sua dignidade humana restituída, a qual pode ser ajudada pelo cumprimento das legislações no meio social, tanto pela sociedade civil quanto pelos órgãos públicos e profissionais da saúde. Neste contexto, estão os portadores de HIV/AIDS enfrentando as dificuldades na saúde pública, uma vez que o Poder Público acaba não conseguindo suprir todas as demandas, o que se deve à má gestão dos recursos, ausência de coordenação, fraudes e corrupção, agravando a situação crítica do Sistema Único de Saúde.

\subsection{ATUAÇÃO DO ENFERMEIRO NA QUALIDADE DE VIDA DE PACIENTES COM HIV/AIDS}

No contexto da AIDS, o enfoque no conceito de qualidade de vida refere-se a um movimento que deve ser priorizado tanto pelo paciente quanto pela sua família e equipe 
médica, pois para a promoção da qualidade de vida não é necessário apenas o controle de sintomas, mas, também uma redução da mortalidade e aumento da perspectiva de vida (OLIVEIRA et al., 2015).

Ao realizar as discussões sobre qualidade de vida em um sentido amplo, é necessário entendê-la como um processo dinâmico e em constante mudança, que inclui a contínua interação entre o indivíduo e o meio ambiente. As condições constituem fatores que influenciam a avaliação interna da qualidade de vida, e os baixos níveis de escolaridade e renda referem-se a condições de vida instáveis, vulnerabilidade social, cultural e econômica, dificuldades de acesso aos serviços médicos e falta de compreensão do seu estado de saúde e tratamento, que afetam negativamente a avaliação da qualidade de vida (PEREIRA et al., 2016).

No contexto da vulnerabilidade de indivíduos e sociedades ao HIV, são muito importantes as estratégias e políticas sociais que visam a salvaguarda de direitos e a integração desses grupos sociais na sociedade de forma autônoma e de respeito às diferenças. Nesse sentido, a finalidade da criação de alguns instrumentos é mensurar a complexidade envolvida na avaliação da qualidade de vida (OLIVEIRA et al., 2015).

Devido à sobrecarga física e mental, aos conflitos de trabalho e à necessidade de conviver com os pacientes, a enfermagem é considerada uma ocupação estressante. As equipes envolvidas no atendimento a esses pacientes precisam ser especialmente preparadas para fornecer suporte físico e mental para ajudá-los a superar todos os impactos. Não se trata apenas de enfermagem, mas de estratégias que podem melhorar e mudar a vida dos pacientes após todas as fases da pandemia do HIV/AIDS (NETO et al., 2019).

Outra competência importante é da equipe é o aconselhamento, inclui o três momentos: suporte emocional para o paciente, avaliação de risco e suporte educacional. Esses componentes estão inter-relacionados e os profissionais de saúde devem estar preparados para cumpri-los de forma eficaz. Consulta é qualquer relação de confiança e diálogo entre profissionais e clientes, baseada na escuta e na comunicação clara e objetiva, de forma que os indivíduos se tornem alvos de prevenção e autocuidado (MARQUES et al., 2020). 
Destaca-se ainda, a adesão à terapia antirretroviral pode trazer uma contribuição significativa de diversas formas, podendo não só melhorar a qualidade de vida dessas pessoas, mas também reduzir a taxa de mortalidade causada pela doença. Principalmente em áreas relacionadas a níveis independentes. Embora essa distinção não seja feita, é importante enfatizar que mesmo que esteja recebendo tratamento e obtendo acesso a saúde por meio da terapia antirretroviral, supondo que, em comparação com indivíduos assintomáticos, os sintomáticos possam ter mais complicações, desconforto e fraqueza física.

Daí a relevância do enfermeiro para o processo de conscientização, neste cenário destaca-se o papel do enfermeiro que representa o maior número de profissionais da equipe de saúde e atua desde o primeiro caso de AIDS no Brasil. O enfermeiro deve ser treinado para prestar um atendimento humanizado, integral e personalizado, com base no conhecimento científico e na Sistematização da Enfermagem (SAE) baseada em PVHIV (MAIA; REIS JÚNIOR, 2019).

Assim, as equipes de enfermagem, em especial as enfermeiras, têm sido capacitadas na prevenção, tratamento e reabilitação em saúde, elemento essencial no acompanhamento dessas pessoas, auxiliando na adesão ao tratamento e melhorando sua qualidade de vida.

\section{CONCLUSÕES}

Conclui-se que a qualidade de vida dos Portadores de HIV/AIDS depende de uma série de fatores correlacionados à manutenção e prestação de uma saúde adequada, levando em consideração que o enfermeiro atua de forma ativa de acordo com as regras éticas e técnicas.

Destaca-se que as pessoas infectadas pelo HIV/AIDS devem ter seu tratamento e medicamento garantido pela saúde pública, ser respeitado na sua privacidade pela sociedade e pelos profissionais de saúde que devem ser éticos, justos e autônomos em conceder igualdade e dignidade humana aos pacientes, para que estes sejam dignos em seus trabalhos e no meio social mesmo sofrendo de uma patologia crônica.

Embora, existam na atualidade várias legislações que garantem os direitos e deveres dos Portadores de HIV/AIDS, estes ainda não usufruem plenamente destas garantias constitucionais por ser o Brasil um país populoso e com isso a grande demanda de 
patologias a serem atendidas e sustentadas pelo Sistema Único de Saúde (SUS), o qual sofre com más gestões políticas e econômicas, o que torna o sistema ineficaz e não tão robusto como é em sua teoria.

Todavia, o SUS ainda é uma referência mundial por ser um sistema de princípios universal, igualitário, gratuito e integral, diferenciando-se das demais nações do planeta terra que ainda não conta com uma ideia tão abrangente dos direitos fundamentais ou humanos em prol dos cidadãos na efetivação da qualidade de vida. Por fim, nesta engrenagem estão os profissionais da saúde, enfermeiros (as) e médicos (as), que precisam estar enquadrados nos princípios da bioética.

Assim, para contribuírem para a existência da qualidade de vida daqueles que estão acometidos pelo mal da AIDS que ainda não tem cura, mas que possui medicamentos paliativos e ações humanas que podem ajudá-los a viverem mais e com dignidade, que são: amor, respeito, empatia, igualdade, doação e vivência das leis em prol do bem-estar físico, emocional e psicossocial dos Portadores de HIV/AIDS.

\section{REFERÊNCIAS}

BRANDÃO, B. M. G. M; PEREIRA, V. M. A. O; GÓIS, A. R. S; SILVA, C. R. L; ABRÃO, F. M. S. Representações sociais da equipe de enfermagem perante o paciente com HIV/AIDS: uma revisão integrativa. Revista de Enfermagem da UFPE online, v. II, n. 2, p. $625-633.2017$.

BRASIL. Ministério da Saúde. Secretaria de Vigilância em Saúde - Programa Nacional de DST e AIDS. Diagnóstico laboratorial da infecção pelo HIV. Recomendações para terapia antirretroviral em adultos e adolescentes infectados pelo $\mathrm{HIV}, 2017$.

BRASIL. Ministério da Saúde. Secretaria de Vigilância em Saúde. Departamento de vigilância epidemiológica. Doenças infecciosas e parasitárias- Guia de Bolso. $8^{\circ}$ Ed. Brasilia-DF. p.444. 2019.

BRASIL. Ministério da Saúde. Secretaria de Vigilância em Saúde. Departamento de DST, Aids e Hepatites Virais. Relatório de monitoramento clínico do HIV. Brasília - DF. p.94., 2017. 
COSTA, T. L; OLIVEIRA, D. C; FORMOZO, G. A. O setor saúde nas representações sociais do HIV/Aids e qualidade de vida de pessoas soropositivas. Revista da Esc. Anna Nery, v. 19, n. 3, p. 475-493. 2015.

HIPOLITO, R. L; OLIVEIRA, D. C; COSTA, T. L; MARQUES, S. C; PEREIRA, E. R; GOMES, A. M. T. Qualidade de vida de pessoas convivendo com HIV/aids: relação temporal, sócio demográfica e perceptiva da saúde. RLAE- Revista Latina Americana de Enfermagem, v. 5, n. 7, p. 55-70. 2017. DOI: 10.1590/1518-8345.1258.2874.

MAIA, É. C. A; REIS JÚNIOR, L. P. Modos de enfrentamento do HIV/AIDS: direitos humanos, vulnerabilidades e assistência à saúde. Revista NUFEN, v.II, n.I, p.54-44.2019. DOI: http://dx.doi.org/ro.26823/RevistadoNUFEN.voliı.noorensaio48.

MARQUES, S. C; OLIVEIRA, D. C; CECILIO, H. O. M; SILVA, C. P; SAMPAIO, L. A; SILVA, V. X. P. Avaliação da qualidade de vida de pessoas vivendo com HIV/AIDS: revisão integrativa. Revista Enfermagem UERJ, v. 28, n. 4, p. 34-52. 2020. DOI: https://doi.org/Io.12957/reuerj.2020.39144.

MARTINS, A; MONTEIRO, F. IALHO, R; CANAVARRO, M. C; PEREIRA, M. Qualidade de vida na infeção VIH: perfis segundo o modo de transmissão. Revista de

Psicologia Saúde \& Doenças, v.17, n. I, p. 7-14. 2016.

NETO, C. M; PIRES, E. M. C; BRITO, C. S; BESERRA, O. L. M. G; SILVA JÚNIOR, J. F; MOTA, J. V; CALDAS, R. T. J. Qualidade de vida no contexto de paciente com HIV/AIDS: Um estudo comparativo. Revista Saúde e Pesquisa da UNICEUSMAR, v.I2, n.2, p. 55-78. 2019. DOI: https://doi.org/10.17765/2176-9206.2019vi2n2p333-34I.

OLIVEIRA, G. A. L. O que as pesquisas revelam sobre a saúde no Brasil. Revista Inovar, v. 5, n. 34, p. I-407. 2019.

OliveirA, M. R. M; DELDUQUE, M. C; SOUSA, M. F; MENDONÇA, A. V. M. Judicialização da saúde: para onde caminham as produções científicas? RevistaSaúde em debate, v. 39, n. 105, p. 525-535. 2015. DOI: https://doi.org/10.1590/o103-110420151050002019. PEREIRA, L. A; FEITOSA, M. C; SILVA, G. R. F; LEITE, I. R. L; SILVA, M. E; SOARES, R. D. M. Pacientes com HIV/Aids e risco de ulcera: demandas de enfermagem. Revista Brasileira de Enfermagem, v. 69, n. 3, p. 77-89. 2016. 